\title{
Measurements of millimeter wave test structures for high speed chip testing
}

\author{
Timothy De Keulenaer, Yu Ban and Guy Torfs \\ INTEC/IMEC, Ghent University \\ Ghent, Belgium
}

\author{
Stefaan Sercu, Jan De Geest \\ FCI (S. Sercu now with Samtec) \\ www.fci.com
}

Johan Bauwelinck

INTEC/IMEC, Ghent University

Ghent, Belgium

\begin{abstract}
This paper presents the frequency domain characterization of very high bandwidth connectorized traces and a millimeter wave rat race coupler. These connectorized differential grounded coplanar waveguide traces, essential for the testability of high speed integrated circuits, have a measured flat frequency response up to $67 \mathrm{GHz}$ which indicates correct connector footprint and transmission line design. The differential traces narrow down to a chip scale pitch of $150 \mu \mathrm{m}$ allowing direct flip chip connections. This enabling the testing of millimeter wave integrated circuits without the need for probing. Furthermore, a $50 \mathrm{GHz}$ rat race coupler was fabricated to generate a differential clock from a single ended clock source.
\end{abstract}

\section{INTRODUCTION}

At high datarates, inter-chip electrical communication over standard traces becomes challenging due to excessive frequency dependent channel attenuation causing large amounts of inter-symbol interference (ISI). Combined with impedance mismatches, a very challenging environment for high speed and high bandwidth communication is created. When testing high speed communication chips, care must be taken in the design of the test board to ensure measurements reflect the chip performance, and not the connectorized test board.

High bandwidth integrated circuit designs face a variety of technical dificulties. First of all, there are the functional and matching requirements, which in the presence of layout parasitics, ESD protection mechanisms and process limitations, can be hard to reach. But, after fabrication, a second problem arises: testing a chip in real life circumstances requires it to be mounted on a board, using either bondwires, a flip chip process or some other kind of packaging. Typically direct chipon-board flip chip assembly (without interposer) adds the least amount of parasitic inductance and capacitance, making it the preferred method for high speed/high bandwidth chip-to-board interconnects with bandwidths above $50 \mathrm{GHz}$.

However, to be able to flip a chip directly on a board, the pitch of the traces on the board and the bondpads on the chip need to align. This clarifies the need of a fine pitched, differential grounded coplanar waveguide (GCPW) structure such as shown in Fig. 1. On the other side of this transmission line structure, single ended connectors are used to connect the test board to high speed test and measurement equipment like a sampling scope, vector network analyser or BER tester.

Furthermore, high speed chips typically need a differential clock to synchronize data to the test and measurement equipment, unless an on chip CDR or balancing circuit is available but this adds complexity and consumes scarce chip real estate. Providing a differential clock at high frequencies requires a single ended to differential converter as most millimeter wave frequency generators only provide a single ended output. To convert this single ended output to a differential clock a modified rat race coupler was designed and measured to work around $50 \mathrm{GHz}$.

Section II describes the design as well as the sizing of a GCPW transmission line and a rat race coupler. In section III, different test structures are defined and the measurements of these test structures and the rat race coupler are discussed. The paper will end with a conclusion on how to select the right connectors and design transmission lines for testing high speed chips on a board.

\section{DESIGN OF MILLIMETER WAVE TEST STRUCTURES}

A high speed test board typically consists of coupled transmission lines starting at the chip, routed to connectors, together with some lower speed control signals and power and ground connections. The most challenges are found in the coupled transmission lines starting from the IC bondpad pitch and tapering out to a reasonable dimension for loss and manufacturing tolerances. Further on, the coupled traces are split into 2 single transmission lines and connected out using a $50 \Omega$ connector. In Fig. 1 a close up is shown of a typical high speed test interconnection.

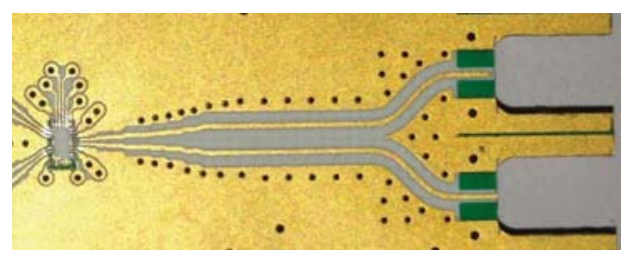

Fig. 1: Typical high speed test interconnection, traces taper out from chip pitch to the connector footprint. In this case a miniSMP footprint is shown.

\section{A. Coupled GCPW design}

To calculate the impedance of a coupled grounded coplanar waveguide structure a 2D impedance calculator developed by the Ghent University INTEC EM group was used [1]. This tool allows one to draw any number of dielectrics and conductors and calculates the differential and common mode impedance 
of 2 single conductors given the dielectric constants and metal thickness, which can be found in the material datasheet or in the manufacturer documentation. A structure as shown at the top of Fig. 2 is drawn and calculated.

The differential impedance is designed to be $100 \Omega$. To connect the chip directly on the traces a pitch of $150 \mu \mathrm{m}$ is required. To manage this within manufacturing possibilities the differential impedance will deviate from $100 \Omega$ as the gap $(\mathrm{G})$ and the clearance (C) are equal to the minimum manufacturable clearance of $70 \mu \mathrm{m}$ and traces (W) are sized to be $80 \mu \mathrm{m}$, which for the $221 \mu \mathrm{m}$ RO4003C with $30 \mathrm{um}$ of plated top metal results in a differential impedance of $120 \Omega$. Further on, the traces are tapered out by a concatenation of tapers to realize the $100 \Omega$ differential impedance and to reduce the influence of manufacturing tolerances. It should be noted that the common mode impedance varies across the concatenation of tapers.
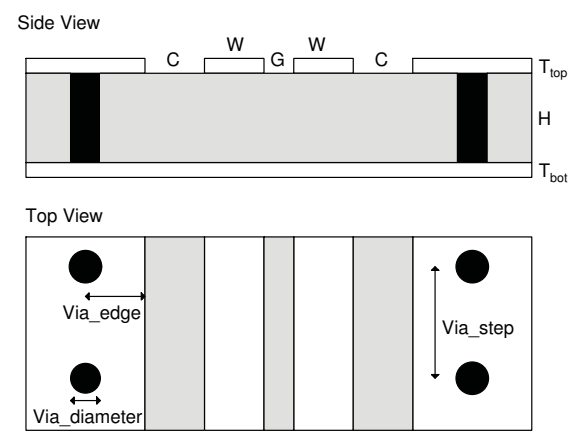

Fig. 2: Side and top view of a coupled grounded coplanar waveguide with design parameters: W (width), G (gap), C (clearance), $\mathrm{H}$ (height), via size, via spacing and distance between vias and traces. $T_{t o p}$ and $T_{b o t}$ are respectivly the top metal thickness including plating and the bottom metal thickness.

As explained in [3] the via spacing, via distance towards the edge of the ground plane and the via diameter have an influence on the bandwidth of the trace. Typically, reducing these distances will improve the bandwidth. Furthermore, the designed test structures use a double via row as advised in the Rosenberger $1.85 \mathrm{~mm}$ reference footprint [4]. The length of the different tapers is always chosen to be equal to the distance between the vias for layout reasons.

\section{B. Coupled to uncoupled GCPW splitter}

To connect the coupled transmission lines to measurement equipment it needs to be split into two single ended transmission lines so that a connector can be mounted at the end of these transmission lines. The dimensions of the coupled and uncoupled coplanar waveguide are used as a starting point of the coupled to uncoupled GCPW splitter. These are connected together in a smooth way to maximize the bandwidth of the transition, the least deviation from a $100 \Omega$ differential line. As parameters we took the angle $(\alpha)$ and the distance between the single ended lines as shown in figure 3. Optimizing $\alpha$ with

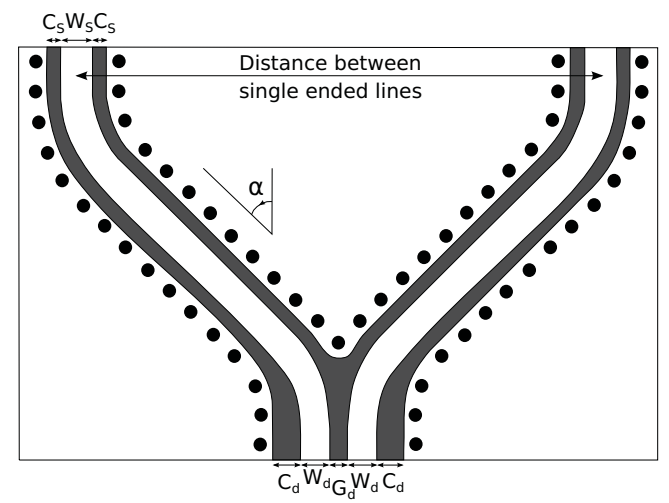

Fig. 3: Coupled to uncoupled GCPW splitter on pcb board. $C_{d}, W_{d}$ and $G_{d}$ are the parameters defining the differential impedance and $C_{s}$ and $W_{s}$ define the impedance of the uncoupled lines.

a fixed distance led to a maximum bandwidth at an angle of around $60^{\circ}$, which resulted in a single ended to differential conversion with a bandwidth of over $100 \mathrm{GHz}$ in simulation.

\section{Footprint design}

In the test structures 2 types of screw on connectors are tested. The Southwest Microwave $2.4 \mathrm{~mm}$ end launch connector and the Rosenberger $1.85 \mathrm{~mm}$ angle launch connector. The footprint design of a connector has a significant influence on the bandwidth of the system. The footprints of both connectors were optimized in CST MWS starting from the footprint advised by the manufacturer [4][5] with a self built connector model. Optimizations were based on TDR analysis as discussed in [3].

\section{Rat race coupler design}

Fabricating traditional rat race couplers for high frequencies becomes a great challenge as a $70.7 \Omega$ circle with a radius of $R=\frac{3 \lambda}{4 \pi}$ is needed [6]. However, as shown in Fig. 4, it is possible to increase the radius of the circle and keep the same differential relationship at the outputs by adding $\frac{\lambda}{2}$ to the short paths and $\frac{3 \lambda}{2}$ to the long path. This results in a radius of $R=\frac{9 \lambda}{4 \pi}$. As a consequence, this rat race coupler also works at $\frac{9 \lambda_{1}}{4 \pi}=\frac{3 \lambda_{2}}{4 \pi}$, one third of the design frequency.

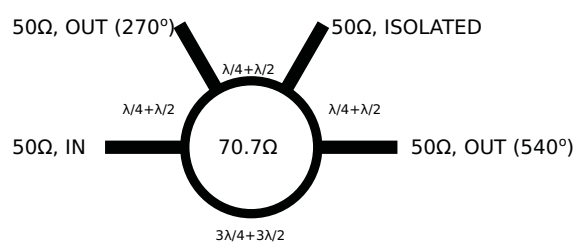

Fig. 4: Topology of the proposed rat race coupler, half wavelength segments are added to increase the size for improved manufacturability.

\section{MEASUREMENTS OF HIGH SPEED TEST BOARDS}

The design methods described above were put into practice on a board design with various test structures fabricated by 
Wrekin Circuits. On the test boards multiple structures were put together to test the different connectors and material losses. The boards were produced on a gold plated Rogers RO4003C substrate to resemble a test board for flip chip or wire bond assembly.

All measurements were done with a 4 port $67 \mathrm{GHz}$ Agilent PNA-X.

\section{A. Material characteristics}

The different test structures were fabricated on a $221 \mu \mathrm{m}$ Rogers RO4003C substrate cladded with $17 \mu \mathrm{m}$ LoPro foil (LoPro) as well as on a $203 \mu \mathrm{m}$ Rogers RO4003C substrate cladded with $17 \mu \mathrm{m}$ electrodeposited copper foil (NoLoPro). As the interest goes to bandwidths above $50 \mathrm{GHz}$, the loss is compared at $50 \mathrm{GHz}$ as shown in Fig. 5 and Fig. 6. It is clear

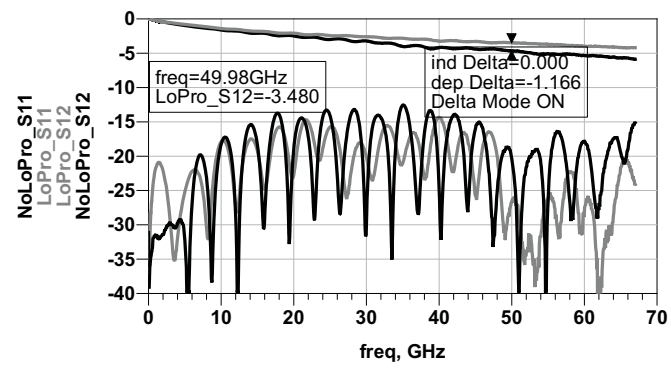

Fig. 5: Measurements of a $26 \mathrm{~mm}$ single ended trace with $1.85 \mathrm{~mm}$ connectors.

that for a single ended transmission line the loss at $50 \mathrm{GHz}$ on the LoPro material is around $1.1 \mathrm{~dB}$ per centimeter and is above $1.4 \mathrm{~dB}$ per centimeter on the NoLoPro material. A summary is given in table I.

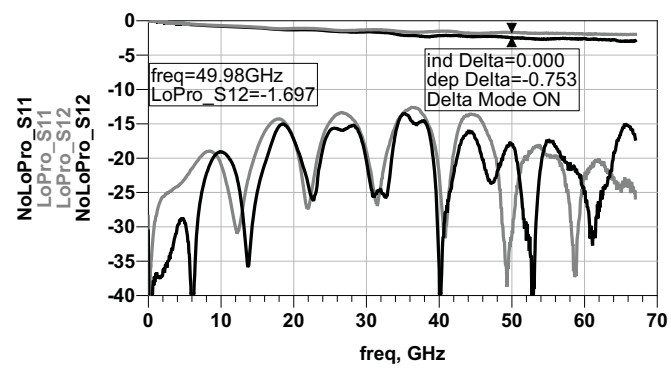

Fig. 6: Measurements of a $10 \mathrm{~mm}$ single ended trace with $1.85 \mathrm{~mm}$ connectors.

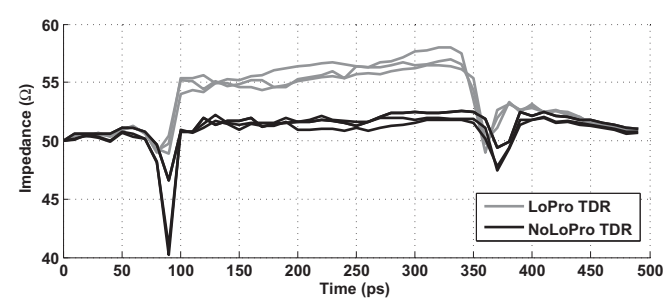

Fig. 7: TDR measurements of $26 \mathrm{~mm}$ traces.

\begin{tabular}{c|c|c|c} 
loss at 50GHz $(\mathrm{dB})$ & $10 \mathrm{~mm}$ & $26 \mathrm{~mm}$ & loss per $\mathrm{cm}$ \\
\hline LoPro & 1.70 & 3.48 & 1.1 \\
\hline NoLoPro & 2.45 & 4.65 & 1.4 \\
\hline loss at 30GHz $(\mathrm{dB})$ & $10 \mathrm{~mm}$ & $26 \mathrm{~mm}$ & loss per $\mathrm{cm}$ \\
\hline LoPro & 1.32 & 2.60 & 0.8 \\
\hline NoLoPro & 1.90 & 3.22 & 0.83
\end{tabular}

TABLE I: Substrate loss at $50 \mathrm{GHz}$ and at $30 \mathrm{GHz}$ calculated from a $26 \mathrm{~mm}$ trace and a $10 \mathrm{~mm}$ trace using $1.85 \mathrm{~mm}$ connectors.

\begin{tabular}{c|c} 
loss at $30 \mathrm{GHz}(\mathrm{dB})$ & $30.4 \mathrm{~mm}$ trace \\
\hline LoPro & 3.5 \\
\hline NoLoPro & 4.5
\end{tabular}

TABLE II: Loss at $30 \mathrm{GHz}$ for $30.4 \mathrm{~mm}$ trace on LoPro and NoLoPro substrate using $2.4 \mathrm{~mm}$ connectors.

The impedance of the line is mainly determined by the dielectric constant $\left(\epsilon_{R}\right)$ and the effective dimensions of the trace. Fig. 7 illustrates that the TDR impedances are about $55 \Omega$ for the LoPro and $52 \Omega$ for the NoLoPro material. Carefull measurement of the lines show a reduction of approximately $10 \%$ in width compared to the designed value, within manufacturer tolerances but resulting in this impedance rise. Multiple samples of each trace were measured and Fig. 7 shows the small sample variation. The design dimensions for the LoPro and NoLoPro material were kept the same, however the NoLoPro material is a bit thinner and has a slighly higher $\epsilon_{R}$ which results in the lower impedances measured. To characterize the losses of the material up to $67 \mathrm{GHz}, 1.85 \mathrm{~mm}$ connectors were connected to 2 different line lengths, $26 \mathrm{~mm}$ and $10 \mathrm{~mm}$. This results in the losses shown in table I.

\section{B. Connector characteristics}

The screw-on connectors are Southwest Microwave $2.4 \mathrm{~mm}$ end-launch connectors and Rosenberger $1.85 \mathrm{~mm}$ angle mount connectors. Both connectors show very clean results. The insertion loss in the $1.85 \mathrm{~mm}$ connector is about $0.3 \mathrm{~dB}$ for the LoPro material and $0.5 \mathrm{~dB}$ for the NoLoPro material at $50 \mathrm{GHz}$, as can be calculated from table I. At $30 \mathrm{GHz}$, the losses of both connectors are comparable.

The loss in the $2.4 \mathrm{~mm}$ connector is about $0.5 \mathrm{~dB}$ for the LoPro material and $1 \mathrm{~dB}$ for the NoLoPro material at $30 \mathrm{GHz}$ as can be calculated from table II. The extra loss on the NoLoPro boards is likely to be caused by a larger capacitive drop at the connector footprint, as illustrated in Fig. 7 for a $26 \mathrm{~mm}$ trace.

\section{Bandwidth of differential lines}

The coupled tapered traces measured in this paper consisted of a multi-stage linear taper to go from the IC bondpad pitch of $150 \mu \mathrm{m}$ to a comfortable pitch (to reduce the influence of manufacturing tolerances) as shown in Fig. 8. In simulation an ideal taper was also analysed by optimizing the taper shape using the tool mentioned in subsection II-A. The simulations did not show a difference between the multi stage linear taper and the ideal taper below $100 \mathrm{GHz}$, the maximum simulation frequency, because the length of the individual linear tapers is 


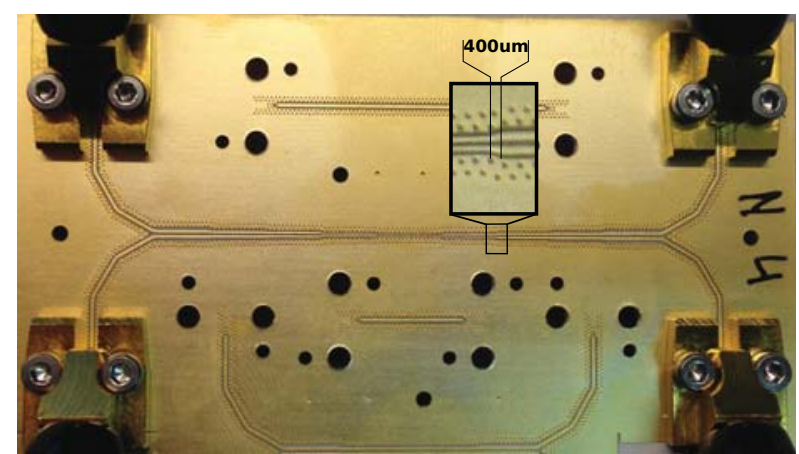

Fig. 8: Photograph of the measured coupled trace tapering to a pitch of $150 \mu \mathrm{m}$. Measurement results are shown in figure 9 .

small compared to the frequency of operation and only shows a minor deviation from the ideal $100 \Omega$ differential impedance.

The loss measured at $50 \mathrm{GHz}$ of the $7.5 \mathrm{~cm}$ tapered trace is almost $11 \mathrm{~dB}$, however the trace loss is smooth and thus can easily be compensated.

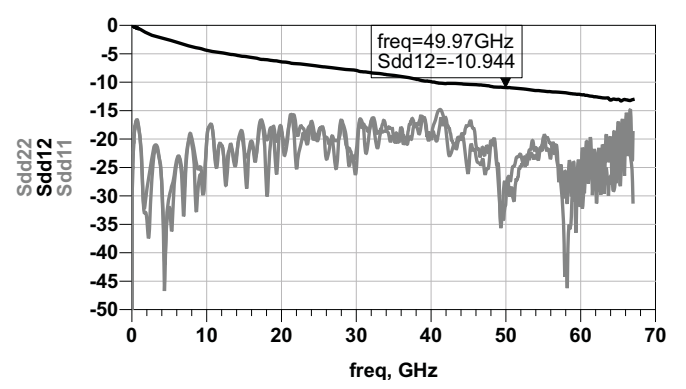

Fig. 9: Measurement of the coupled tapered trace shown in Fig. 8 , both ends are connected to $1.85 \mathrm{~mm}$ connectors. The center trace pitch is $150 \mu \mathrm{m}$ corresponding to a chip-scale pitch. The total trace length is about $7.5 \mathrm{~cm}$.

\section{Rat race coupler}

The rat race coupler shown in Fig. 10 consists of a $275 \mu \mathrm{m}$ wide center circle with a radius of $2800 \mu \mathrm{m}$, which corresponds to $70.7 \Omega$ on the Rogers RO4003C $221 \mu \mathrm{m}$ LoPro substrate. The connecting traces are $375 \mu \mathrm{m}$ wide $50 \Omega$ traces with a Southwest Microwave $2.4 \mathrm{~mm}$ connector launch. The

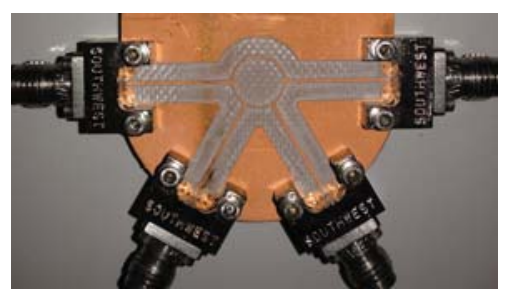

Fig. 10: Photograph of the measured rat race coupler.

measured results are shown in Fig. 11. It is clear that the rat race coupler works around $50 \mathrm{GHz}$. The amplitude difference is below $1 \mathrm{~dB}$ and the phase difference is within $10 \%$ of the designed $180^{\circ}$ from $47 \mathrm{GHz}$ to $52 \mathrm{GHz}$.

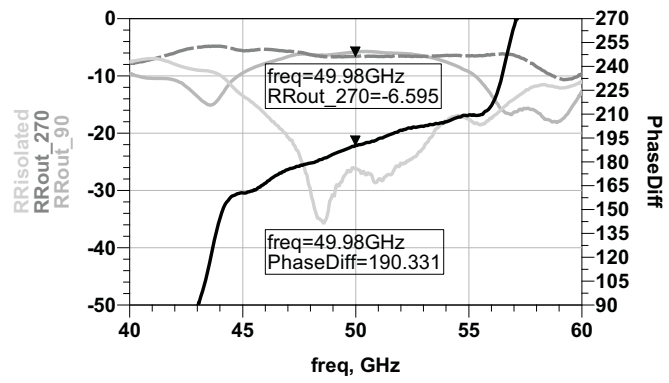

Fig. 11: Measurement results the $50 \mathrm{GHz}$ rat race coupler shown in Fig. 10.

\section{CONCLUSION}

In this paper, broadband measurements up to $67 \mathrm{GHz}$ of different high bandwidth test structures were presented. These structures show that not only probe testing is possible with millimeter wave integrated circuits, but also show the possiblity of mounting the devices on board, thus enabling system experiments in a more complex setup. The difference in loss between Rogers RO4003C LoPro and NoLoPro materials, $1.1 \mathrm{~dB}$ and $1.4 \mathrm{~dB}$ per centimeter at $50 \mathrm{GHz}$ respectively, was shown, as well as a comparison between screw connectors from Rosenberger and Southwest Microwave. Coupled traces, tapered down to $150 \mu \mathrm{m}$ pitch, allowing to directly flip a chip on the traces and a $50 \mathrm{GHz}$ rat race coupler to convert a single ended clock into a differential clock, prove the feasibility of a high speed, board mounted measurement setup.

At $50 \mathrm{GHz}$ and above, LoPro material has a clear advantage over NoLoPro material because the loss per centimeter is about $25 \%$ lower. At $30 \mathrm{GHz}$ and below, however, there was hardly any difference in loss between the two materials. Comparing the $1.85 \mathrm{~mm}$ Rosenberger connector to the $2.4 \mathrm{~mm}$ Southwest Microwave connector showed that the $2.4 \mathrm{~mm}$ connector has about two times the insertion loss at $60 \%$ of their maximum frequency.

\section{ACKNOWLEDGEMENT}

The authors would like to thank the Agency for Innovation by Science and Technology in Flanders (IWT) and the INTEC EM group for their 2D impedance calculator.

\section{REFERENCES}

[1] Demeester, T. et al., Daniël, Accurate study of the electromagnetic and circuit behavior of finite conducting wedges and interconnects with arbitrary cross-sections, IEEE EPEPS, 2010

[2] Simons, Rainee N, Coplanar waveguide circuits, components, and systems, J. Wiley, 2004

[3] Rosas, B., Optimizing Test Boards for $50 \mathrm{GHz}$ End Launch Connectors: Grounded Coplanar Launches and Through Lines on 30-mil Rogers RO4350B with Comparison to Microstrip, Southwest Microwave Inc., Tempe, AZ, 2007

[4] Rosenberger Corp., Recommended PCB layout $1.85 \mathrm{~mm}$ connector (MB_389), 2012

[5] Southwest Microwave Inc, 2.40mm JACK (FEMALE) END LAUNCH CONNECTOR (1492-03A-5), 2012

[6] Chang, K. et al., Microwave ring circuits and related structures, J. Wiley, 2004 